\title{
Optical Energy-Constrained Slot-Amplitude Modulation for Dimmable VLC: Suboptimal Detection and Performance Evaluation
}

\author{
Thai-Chien Bui ${ }^{\circledR}$, Member, IEEE, Ravinder Singh ${ }^{\circledR}$, Member, IEEE, Timothy O’Farrell ${ }^{\circledR}$, Senior Member, IEEE, \\ and Mauro Biagi ${ }^{\circledR}$, Senior Member, IEEE
}

\begin{abstract}
Energy-constrained slot-amplitude modulation (ECSAM) enables light dimming, eliminates light flicker and constrains the peak optical power while providing robust communication links. However, the complexity of the maximum-likelihood (ML) based ECSAM receiver increases exponentially with required spectral efficiency. This paper provides a comprehensive performance evaluation of ECSAM for the indoor visible light communication (VLC) channel with multipath propagation under realistic illumination constraints and imperfect channel estimation. A sub-optimal receiver that employs a slot-by-slot detection algorithm followed by a slot-correction mechanism for reducing the receiver complexity is proposed. Additionally, the method for optimal selection of parameters when designing the signal waveform is presented. The analytical upper bound on the symbol error rate of ECSAM is derived using the union-bound technique. The results show that the error performance of the sub-optimal receiver are comparable to that of the optimal ML receiver. Compared with conventional power or bandwidth efficient VLC modulation techniques such as multiple pulse position modulation (MPPM) and pulse amplitude modulation (PAM), ECSAM provides complete flexibility in modifying the signal constellation for a desired dimming level to maximise the spectral efficiency and provide a robust bit error rate performance especially in the multipath propagation channel induced intersymbol interference.
\end{abstract}

Index Terms-Visible light communication, slot-amplitude modulation, dimming control, multipath propagation, suboptimal detection.

\section{INTRODUCTION}

$\mathbf{O}$ VER recent decades, the demand for ubiquitous connectivity and high capacity data services has been growing rapidly due to the high demand for multimedia, cloud-based

Manuscript received September 2, 2019; revised February 15, 2020, July 17, 2020, and October 18, 2020; accepted October 26, 2020. Date of publication November 4, 2020; date of current version March 10, 2021. The associate editor coordinating the review of this article and approving it for publication was A. Bletsas. (Corresponding author: Thai-Chien Bui.)

Thai-Chien Bui and Mauro Biagi are with the Department of Information, Electrical and Telecommunication (DIET) engineering, "Sapienza" University of Rome, 00184 Rome, Italy (e-mail: chien.buithai@uniromal.it; mauro.biagi@uniroma1.it).

Ravinder Singh is with the Department of Engineering Science, University of Oxford, Oxford OX1 3PJ, U.K. (e-mail: ravinder.singh@eng.ox.ac.uk).

Timothy O'Farrell is with the Department of Electronic and Electrical Engineering, The University of Sheffield, Sheffield S10 2TN, U.K. (e-mail: t.ofarrell@sheffield.ac.uk).

Color versions of one or more of the figures in this article are available online at https://ieeexplore.ieee.org.

Digital Object Identifier 10.1109/TWC.2020.3034584 services and other content over wireless links. This trend is leading to the drastic increase in the wireless data traffic that the commercially exploited radio-frequency (RF) spectrum can no longer support [1]. Visible light communication (VLC), that employs visible light spectrum from $380 \mathrm{~nm}$ to $750 \mathrm{~nm}$, has been proposed for short-range wireless data transmission as a complementary green technology to existing RF technology, particularly in indoor environments where optical sources such as the light emitting diode (LED) are densely deployed for illumination purposes [2], [3].

The integration of communication with lighting infrastructure faces two main challenges when designing an efficient modulation format. These are flicker mitigation and dimming support, both of which are essential for any illumination system. Light flicker is due to the physiology of the human eye relating to the repetitive change in brightness generated from intensity modulation at low frequency, which is potentially a human hazard [4]. It causes several adverse impacts on human health such as migraines, epileptic seizure and increased incidence of headaches in office workers and, thus, it should be avoided in any lighting system [5]. The other important requirement of a VLC system is the ability to guarantee full light source dimming control for power saving and energy efficiency [6] while efficiently transferring data to users. However, at low dimming targets the signal-to-noise ratio (SNR) is significantly decreased due to the reduced transmit optical power, which might affect the quality-of-service of the communication link. On the other hand, at high dimming targets the average transmit optical power is very high leading to high SNR. However, with the upper-bound limit on peak transmit power the max-min distance of the transmitted power (i.e. the transmitted power dynamic range) is very small resulting in high SNR levels required to correctly estimate the symbol at the receiver. Therefore, advanced modulation schemes should be designed that adapt to the required dimming level and efficiently utilize the available SNR (which increases with increasing dimming) for the highest possible data rate, while avoiding intensity flicker.

One of the simplest modulation techniques is on-off keying (OOK) where compensating symbols are inserted for every data frame to adjust the average intensity of the illumination source [7]. In this case, the bit pattern is no longer random and, therefore, it is possible to have long runs of zeros and/or 
ones within a frame that can change the average illumination level, causing flicker and leading to signal distortion due to a high lower cut-off frequency of the electrical components within the VLC transmitters. Consequently, a symmetric Manchester code is usually used in OOK at the expense of reduced achievable data rate at low and high dimming levels. Another technique with dimming support based on the pulse width modulation (PWM) principle is variable pulse position modulation (VPPM) where each symbol is composed of two slots with variable pulse width, which is varied in accordance with the required dimming levels [7]. Such techniques have the ability to support a full range of dimming with low complexity at the cost of low spectral efficiency (SE), which is always smaller than $1 \mathrm{bit} / \mathrm{s} / \mathrm{Hz}$. Higher order modulation schemes such as multiple pulse position modulation (MPPM) [8], overlapping pulse position modulation (OPPM) [9], expurgated PPM (EPPM) [10], and multi-level MPPM block coding (MLMPPM) [11] have been proposed to increase the SE while having the ability to support various discrete dimming levels. Basically, both MPPM and OPPM are similar to conventional $N$-ary PPM, however, they permit multiple active pulses in any of the $N$ pulse chips of symbols and the dimming level is defined based on the number of active pulse slots in each symbol duration. In contrast, EPPM controls the peak to average power ratio (PAPR) by using the incidence matrix of a symmetric balanced incomplete block design (BIBD). Therefore, the number of dimming levels supported depends on the number of BIBD codes available. Similarly, ML-MPPM is a block coding based modulation scheme, where the code length is the number of pulse slots in each symbol. The weight of each code determines the brightness of the system. The dimming resolution of ML-MPPM depends on the size of the codeword. Another potential solution to mitigate the severe SE degradation caused by light dimming is to use complementary PPM (CPPM) together with a modified version of color shift keying (CSK) as proposed in [12]. In addition, inverse source coding such as Huffman coding has been proposed in [13], [14] where the amplitudes and probability mass functions of the symbols are selected in order to obtain a target dimming level.

Recently, the authors proposed a novel VLC modulation scheme named energy-constrained slot-amplitude modulation (ECSAM) [15] such that both the flicker and dimming issues can be resolved by considering an energy constraint within the duration/period of every macro-symbol, which consists of multiple amplitude modulated slots, referred to as micro-symbols. ECSAM controls both time and amplitude dimensions, which can be interpreted as a smart merger of amplitude (e.g. pulse amplitude modulation - PAM) and position (e.g. PPM) based modulation schemes. The duration of each macro-symbol is set to be less than the human eye's integrating response time, providing equal energy level among all the macro-symbols for flicker-free transmission. In addition to the flicker mitigation, the scheme is able to provide full-range dimming support, meeting illumination requirements. In [16], the principle of ECSAM is applied to spatial domain in place of time domain to increase the spectral efficiency leading to a modified generalized spatial modulation (MGSM) scheme where the space and amplitude dimensions are smartly combined under practical illumination constraints as in ECSAM.

However, the authors have highlighted in the past the issue of receiver complexity of ECSAM when higher spectral efficiency is required [15]. In this work, the authors discuss a method for optimal selection of parameters when designing the ECSAM signal waveform under full dimming control. We propose a sub-optimal receiver for ECSAM to reduce the detection complexity by employing a simple slot-by-slot detection optimized with a slot-correction mechanism based on the constraint of the transmit optical symbol energy. The effect of imperfect channel estimation on the system error performance is also evaluated and discussed. An upper bound on error rate is derived theoretically using the union-bound technique and is verified by extensive simulations. The error performance of ECSAM is compared with conventional modulation formats under both LOS and hybrid channels with inter-symbol interference (ISI) induced by multipath propagation. The investigations find that the proposed sub-optimal receiver can be a low complexity detection format in ECSAM with comparable error performance to optimal ML detection. When fixing the system bandwidth at $75 \mathrm{MHz}$, ECSAM outperforms PAM in terms of error probability especially in a hybrid channel for all the dimming levels required. When comparing at the same bit rate of $100 \mathrm{Mbit} / \mathrm{s}$, dimmable-PAM has lower bit error probability at dimming range from 0.36 to 0.63 for a LOS channel. However, ECSAM provides significantly better error performance in an ISI induced channel.

The organization of the paper is as follows. In Section II, we formulate the considered indoor VLC channel model, explain the constraints in terms of illumination on a VLC system and introduce the ECSAM transmission scheme. In Section III, the theoretical performance analysis of the ECSAM system using optimal and suboptimal receivers is presented. Simulation results and performance comparisons are presented in Section IV. Finally, Section V includes the main conclusions of the paper. Notation: Bold lowercase letters are used for vectors. (. $)^{T}$ denotes vector or matrix transpose. \|. $\mid$ stands for the Frobenius norm. $*$ is used as the convolution operator. $P($.$) and E($.$) represent the probability of an event$ and its expectation, respectively. The term $\left(\begin{array}{l}n \\ k\end{array}\right)$ is used for the binomial coefficient. We use $\lfloor x\rfloor$ and $\lceil x\rceil$ as the largest integer less than or equal to $x$ and smallest integer greater than or equal to $x$, respectively. Furthermore, $\{x \mid f(x)\}$ means the set of all $x$ for which $f(x)$ is true. The cardinality of a set $\mathbf{x}$ is denoted by $|\mathbf{x}|$ while $|x|$ denotes the absolute value of the variable $x$. We use $\langle t\rangle$ for the time averaged value of $t$ and $\delta($.$) for the Dirac delta function.$

\section{SySTEM MODEL}

In this section, fundamental requirements from an illumination perspective are first analyzed as a benchmark, which are used when evaluating the system performance of different modulation schemes. ECSAM principle is detailed taking into account all of the illumination requirements. The indoor optical wireless channel with multipath propagation from reflections is also described for a practical VLC system. 


\section{A. Illumination Constraints for Indoor VLC}

In this subsection, we discuss the practical requirements relating to illumination, which should be considered when designing an efficient modulation scheme for indoor VLC systems. As LEDs are current-driven devices, denote the peak forward current of the LED as $I_{p}$, which is the highest input current that the LED can handle. In general, operating the LED at this peak current will give a shorter operational lifetime and unstable illumination. It is also inefficient since such a current level falls within the LED's non-linear input-output region. Therefore, in practice LEDs are operated at the maximum current level of $I_{\max }$, which is of a lower value (i.e. $I_{\max }<I_{p}$ ). The optical intensity emitted from the LED at this maximum forward current is denoted as $P_{\max }$ (watt). Consequently, when the light intensity of each LED is modulated to carry signal information, there are lower and upper limits, as the first constraint, on the transmit optical intensity signal $x_{i}$ for each LED at the $i$ th instance, which can be expressed as $0 \leq x_{i} \leq P_{\max }$. This limit on the transmitted optical power intuitively restricts the type of modulation formats that can be applied to indoor VLC systems. Furthermore, when designing a communication link to be integrated into a smart indoor lighting system, it is challenging to keep good illumination quality while modulating the light for data transmission.

The second constraint is related to the flicker effect, which is caused by the repetitive change in brightness due to intensity modulation at low frequency. There are two main causes of flicker. First, when each symbol has the same average amplitude, flicker may happen when the modulation frequency is low enough to make the light intensity fluctuations perceivable by the human eye, as in the case of PPM. Second, when light is modulated at high frequency, long runs of uneven symbol amplitudes may cause flicker as in the case of PAM. In all scenarios, a lighting system with light-flicker should be avoided as it has several potential adverse impacts on human health such as migraines, epileptic seizure and increased incidence of headaches in office workers.

The third illumination constraint that needs to be addressed is the full-range and continuous dimming support capability. In scenarios that need a diverse range of lighting, smart light sources should be dimmable to adapt their brightness depending on the environment and application of the lighting for visual comfort and energy conservation. Therefore, dimming can be considered mandatory for LED lighting. Define $z$ $(0<z<1)$ as the dimming ratio, which refers to the reduction in the output optical power of the light source for a meaningful communication, for instance $z=0.75$ means $75 \%$ below the maximum value. Consequently, the dimming constraint of an LED can be expressed as

$$
P_{t}=z P_{\max }
$$

where $P_{t}$ is the average transmit power of the LED. Note that for a meaningful communication, the dimming ratio $z$ has to be larger than 0 and smaller than 1 .

In ECSAM, flickering and dimming constraints are jointly resolved by fixing the total transmit power in each symbol or the symbol energy in the optical domain.

\section{B. ECSAM Symbol Design and Optimization}

This subsection explains the principle of the ECSAM encoder and the optimal selection of parameters when designing the signal waveform. In ECSAM, similar to PPM, the symbol period of $T_{s}$ is partitioned into $N$ equal slots each lasting $T_{p}$ seconds. A slot, during a symbol transmission, can be inactive or active. In each active slot during a symbol transmission, the LED emits an amount of optical power chosen from a set of $M$ possible discrete power levels as $S=\{a, 2 a, \ldots, M a\}$. The amplitude step $a$ can be varied under the lower and upper bound conditions of the transmitted signal power, which are $a>0$ (for active slot) and $M a \leq P_{\max }$, respectively. Assume that the transmit optical power in the time slot $j$ during a symbol transmission is $a \kappa_{j}$ where $\kappa_{j}$ is one of the $M+1$ amplitude indices $\{0,1, \ldots, M\}$. The ECSAM symbol emitted by the LED source during a symbol transmission can be written as

$$
x(t)=a \sum_{j=1}^{N} \kappa_{j} g\left(t-j T_{p}\right),
$$

where $g(t)$ denotes the rectangular pulse-shaping filter function of unit amplitude and duration $T_{p}$. The optical energy per symbol is given as

$$
\mathcal{E}_{s}^{o}=a \sum_{j=1}^{N} \kappa_{j} T_{p} .
$$

When the LED emits maximum optical power $P_{\max }$ for all slots, the maximum energy level measured in every symbol period in the optical domain is then given by $\mathcal{E}_{\max }^{o}=P_{\max } T_{s}$, which corresponds to full illumination or no dimming. In this work, the notations $(.)^{o}$ and $(.)^{e}$ are used for optical domain (at the transmitter) and electrical domain (at the receiver), respectively. In order to dim the lighting level in ECSAM, the transmit optical energy allocated to every ECSAM symbol is fixed at

$$
\mathcal{E}_{s}^{o}=z \mathcal{E}_{\max }^{o}=z P_{\max } T_{s}
$$

where $z$ is the dimming level requested. Substituting (3) into the constraint in (4), we gives

$$
\sum_{j=1}^{N} \kappa_{j}=\frac{z N P_{\max }}{a} .
$$

By fixing the transmit symbol energy for each dimming level, ECSAM completely eliminates the flicker and stroboscopic effects caused by intensity variations between symbols. Furthermore, by considering a small time slot of less than the time reference for the human eye perception $T_{e}$ so that $T_{s}<N T_{e}$, the brightness fluctuation within every symbol is not perceivable by the human eye.

For the sake of simplicity, we denote $\lambda=\sum_{j=1}^{N} \kappa_{j}$, which is essentially the summation of amplitude indices of $N$ slots in a symbol. With $\mathbf{x}=\left[x_{1}, x_{2}, \ldots, x_{N}\right]$ being the transmitted signal vector for a symbol transmission, the set of ECSAM symbol vectors available under the above illumination 


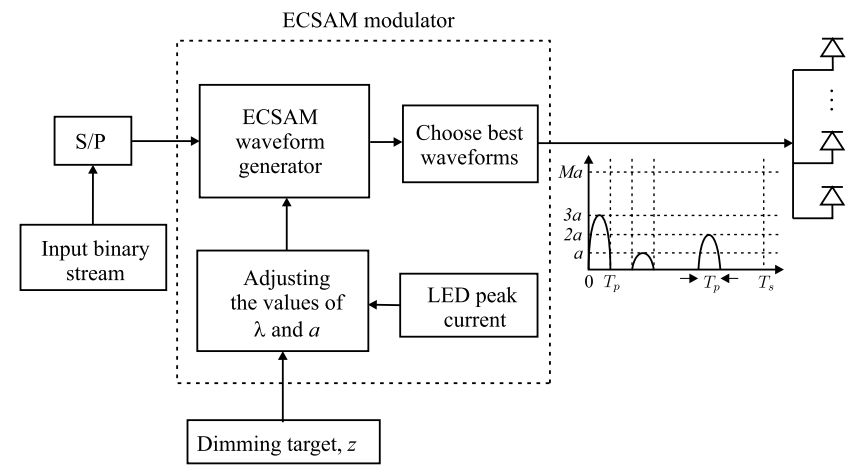

Fig. 1. Block diagram of the proposed ECSAM modulator.

constraints can be written as

$\mathbb{S}_{z, M}^{N}=\left\{\mathbf{x}=a\left[\kappa_{1}, \ldots, \kappa_{N}\right] \mid \lambda=\frac{z N P_{\max }}{a}, 0<a \leq \frac{P_{\max }}{M}\right\}$.

The block diagram of the ECSAM modulator is illustrated in Fig. 1. The constellation size of ECSAM is the cardinality of the set $\mathbb{S}_{z, M}^{N}$ as $\mathcal{Q}=\left|\mathbb{S}_{z, M}^{N}\right|$. In order to calculate $\mathcal{Q}$, consider $\kappa$ as a dice with $M+1$ faces displaying numbers from 0 to $M$, $\kappa_{i}$ is the resultant face of the $i$ th throw of the dice $\kappa$. From (6), $\mathcal{Q}$ can be interpreted as the total number of combinations of $N$ throws of the dice that lead to a fixed (positive) sum of the resultant faces as $\lambda$. To apply this, we redefine the above problem by assuming that the dice has $M+1$ faces numbered from 1 to $M+1$. The sum of the resultant faces from $N$ throws becomes $\lambda+N$. Based on the work in [17], with $q_{\max }=$ $\left\lfloor\frac{\lambda}{M+1}\right\rfloor$, the general formula for the constellation size $\mathcal{Q}$ of ECSAM at dimming level $z$ can be calculated as

$$
\mathcal{Q}=\sum_{i=0}^{q_{\max }}(-1)^{i}\left(\begin{array}{c}
N \\
i
\end{array}\right)\left(\begin{array}{c}
\lambda+N-(M+1) i-1 \\
\lambda-(M+1) i
\end{array}\right) .
$$

It is seen that the constellation size of ECSAM is a function of $\lambda$. Consequently, ECSAM can be considered as an adaptive modulation scheme, which can provide same number of bits per symbol at different dimming levels by changing the amplitude step $a$. The achievable SE $\eta_{\text {ECSAM }}$ of ECSAM with dimming support can be expressed as

$$
\eta_{\mathrm{ECSAM}}=\frac{\left\lfloor\log _{2} \mathcal{Q}\right\rfloor}{N} .
$$

As can be seen in (6), there are multiple waveform sets available at each dimming level satisfying the illumination constraints in ECSAM. Considering a dimming level $z$, the lower and upper bounds of $\lambda$ can be expressed as:

$$
\lceil z M N\rceil \leq \lambda<M N .
$$

The corresponding bounds for $a$ are then given by

$$
\frac{z P_{\max }}{M}<a \leq \frac{z N P_{\max }}{\lceil z M N\rceil} .
$$

Figures 2(a) and 2(b) illustrate the relationship between $a$, $\lambda$ and $\eta_{\text {ECSAM }}$ at different dimming levels for the case of $M=$ 4 and $N=4$ with normalized maximum transmit power of $P_{\max }=1$. The inversely proportional relationship between $a$

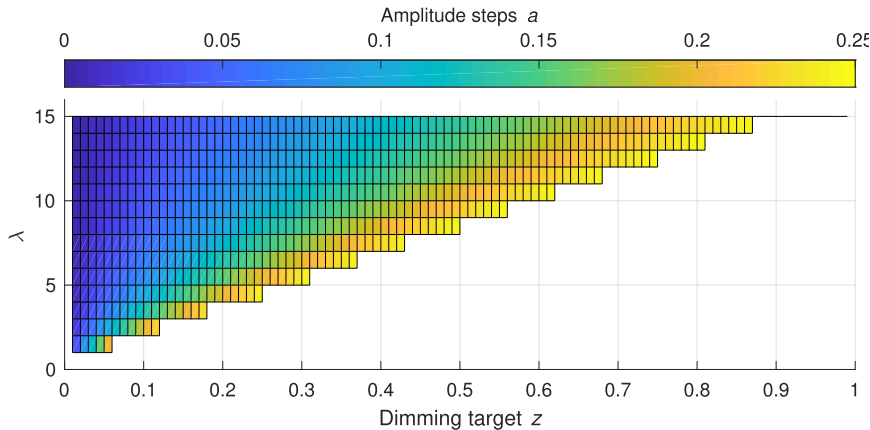

(a) Amplitude steps $a$

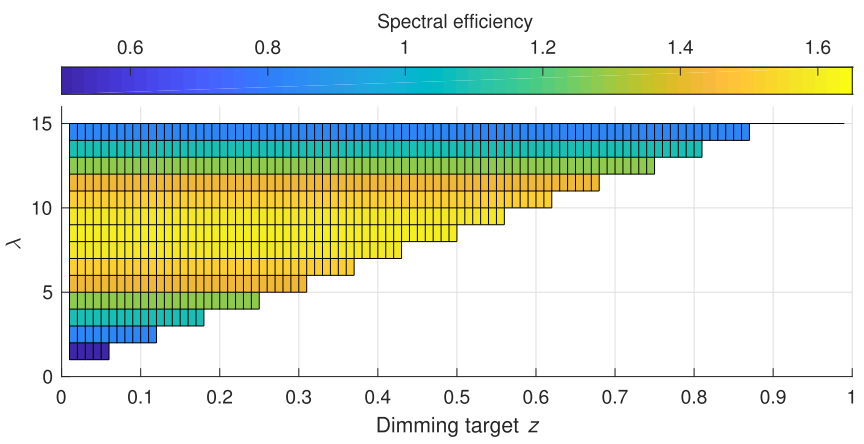

(b) Achievable spectral efficiency

Fig. 2. Visualization of the resultant $\lambda$, the amplitude step $a$ and the achievable spectral efficiency over the full range of dimming for $M=4$ and $N=4$.

and $\lambda$ can be visualized clearly in Fig. 2(a). In terms of SE, it is seen that by fixing $\lambda$, a constant SE can be achieved irrespective of dimming level as depicted in Fig. 2(b). The highest values of SE are obtained at the middle range of $\lambda$ and symmetrically reduce for low and high values of $\lambda$. More specifically, the peak SE achieved is about $1.60 \mathrm{bit} / \mathrm{s} / \mathrm{Hz}$ at $\lambda=8$ for this particular setup. A dimming level $z=0.3$ requires a minimum value of $\lambda=5$, which corresponds to the maximum $a$ of 0.24 and $\eta=1.42 \mathrm{bit} / \mathrm{s} / \mathrm{Hz}$. When the dimming level $z=0.7$ is requested, $\lambda>12$ is required, which corresponds to a maximum $a$ of 0.23 and a SE of less than $1.28 \mathrm{bit} / \mathrm{s} / \mathrm{Hz}$.

ECSAM gives a flexibility in designing the signal waveforms for illumination-constrained VLC. In this work, we focus on minimizing the error rate of the system. It is noteworthy that the amplitude step $a$ has a direct impact on the Euclidean distance between ECSAM waveforms. For optimizing the error performance, the highest value of $a$ should be considered, which corresponds to the lowest $\lambda$ of $\lceil z M N\rceil$. The optimized set of ECSAM symbol vectors at the dimming level $z$ in (6) can be rewritten as

$\mathbb{S}_{z, M}^{N}=\left\{\mathbf{x}=a\left[\kappa_{1}, \ldots, \kappa_{N}\right] \mid \lambda=\lceil z M N\rceil, a=\frac{z N P_{\max }}{\lambda}\right\}$,

where $\kappa_{j}=0,1, \ldots, M$.

Example 1: Consider an indoor VLC system with parameters $M=4, N=4$, and $P_{\max }=1$ watt as in Fig. 2 . In this example, a dimming target of $z=0.25$ is demonstrated. For this setup, the maximum value of $a$ is 0.25 , 
TABLE I

EXAMPle OF 32 MACRO-SYMbol WAVEForms OF ECSAM FOR $z=0.25, M=4, N=4$ AND $P_{\mathrm{MAX}}=1$ watt

\begin{tabular}{|c|c|c|c|c|c|}
\hline & Binary value & $T_{1}$ & $T_{2}$ & $T_{3}$ & $T_{4}$ \\
\hline 0 & 00000 & 0 & 0 & 0 & 1 \\
\hline 1 & 00001 & 0 & 0 & 0.25 & 0.75 \\
\hline 2 & 00011 & 0 & 0.25 & 0 & 0.75 \\
\hline 3 & 00010 & 0.25 & 0 & 0 & 0.75 \\
\hline 4 & 00110 & 0.25 & 0 & 0.25 & 0.5 \\
\hline 5 & 00111 & 0.25 & 0.25 & 0 & 0.5 \\
\hline 6 & 00101 & 0.5 & 0 & 0 & 0.5 \\
\hline 7 & 00100 & 0.5 & 0.25 & 0 & 0.25 \\
\hline 8 & 01100 & 0.75 & 0 & 0 & 0.25 \\
\hline 9 & 01101 & 1 & 0 & 0 & 0 \\
\hline 10 & 01111 & 0.75 & 0 & 0.25 & 0 \\
\hline 11 & 01110 & 0.75 & 0.25 & 0 & 0 \\
\hline 12 & 01010 & 0.5 & 0.25 & 0.25 & 0 \\
\hline 13 & 01011 & 0.5 & 0.5 & 0 & 0 \\
\hline 14 & 01001 & 0.25 & 0.5 & 0 & 0.25 \\
\hline 15 & 01000 & 0.25 & 0.5 & 0.25 & 0 \\
\hline 16 & 11000 & 0.25 & 0.75 & 0 & 0 \\
\hline 17 & 11001 & 0 & 1 & 0 & 0 \\
\hline 18 & 11011 & 0 & 0.75 & 0 & 0.25 \\
\hline 19 & 11010 & 0 & 0.75 & 0.25 & 0 \\
\hline 20 & 11110 & 0 & 0.5 & 0.5 & 0 \\
\hline 21 & 11111 & 0 & 0.25 & 0.5 & 0.25 \\
\hline 22 & 11101 & 0 & 0.25 & 0.75 & 0 \\
\hline 23 & 11100 & 0.25 & 0.25 & 0.5 & 0 \\
\hline 24 & 10100 & 0.5 & 0 & 0.5 & 0 \\
\hline 25 & 10101 & 0.25 & 0 & 0.5 & 0.25 \\
\hline 26 & 10111 & 0.25 & 0 & 0.75 & 0 \\
\hline 27 & 10110 & 0 & 0 & 1 & 0 \\
\hline 28 & 10010 & 0 & 0 & 0.75 & 0.25 \\
\hline 29 & 10011 & 0 & 0 & 0.5 & 0.5 \\
\hline 30 & 10001 & 0 & 0.25 & 0.25 & 0.5 \\
\hline 31 & 10000 & 0 & 0.5 & 0 & 0.5 \\
\hline 32 & - & 0 & 0.5 & 0.25 & 0.25 \\
\hline 33 & - & 0.25 & 0.25 & 0.25 & 0.25 \\
\hline 34 & - & 0.5 & 0 & 0.25 & 0.25 \\
\hline
\end{tabular}

which corresponds to a minimum $\lambda=4$. Therefore, the set of amplitude levels is $S=\{0.25,0.5,0.75,1\}$. From (7), there are 35 possible amplitude combinations that satisfy the dimming and maximum power constraints in this particular ECSAM configuration. All the signal waveforms of the proposed ECSAM modulator at $z=0.25$ are presented in Table I. The Euclidean distance among all combinations was calculated and 32 macro-symbols with the greatest Euclidean distance amongst themselves are selected for signal waveforms. The arrangement maps 5 bits per macro-symbol as shown in Table I.

\section{The Indoor VLC Channel Model}

We consider an indoor VLC system, where the optical wireless channel link between the LED and photodiode (PD) pair is composed of a LOS path and multiple delayed paths reflected off the walls, floor and other indoor objects as depicted in Fig. 3. The total channel impulse response (CIR) including responses from LOS and all reflections due to multipath propagation can be calculated as:

$$
h(t)=\sum_{k=0}^{\infty} h^{(k)}(t)
$$

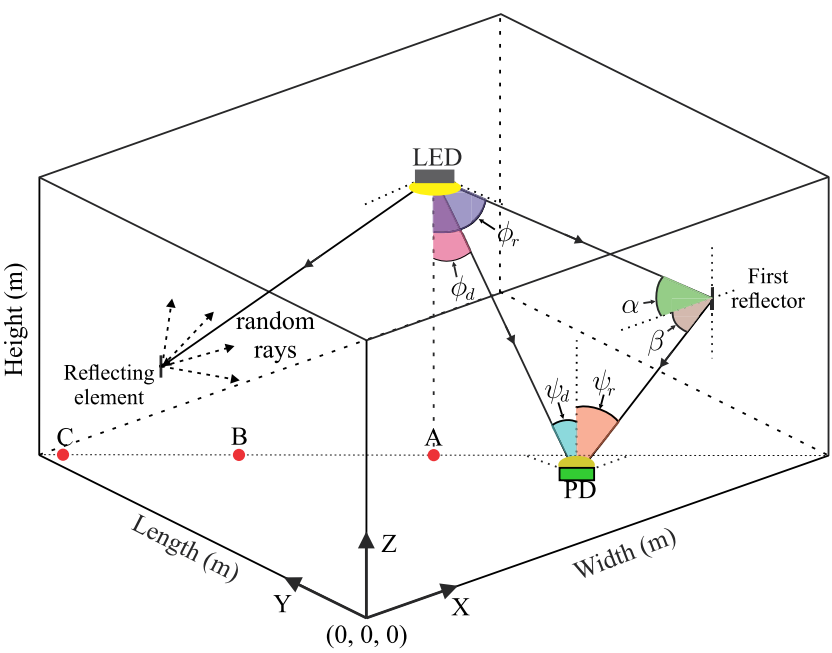

Fig. 3. Room configuration with multipath propagation for channel estimation and system evaluation.

where $h^{(0)}(t)$ is the LOS response which can be easily calculated as in [18], while $h^{(k)}(t)(k>0)$ represents the responses of the $k$ th reflection.

The CIR from multipath propagation in such a hybrid channel environment can be modeled accurately using a combined deterministic and modified Monte Carlo (CDMMC) technique [19]. Each interior wall surface of the room is divided into small reflecting elements of area $\Delta A_{\text {ele }}$. First, the received optical power $P_{\text {ele-receive }}$ at each reflecting element from the LED is calculated. Each reflecting element now becomes a source with emission power $P_{\text {ele-emit }}=\rho_{\text {ele }} P_{\text {ele-receive }}$ where $\rho_{\text {ele }}$ is the reflection coefficient of the element, and the LOS contribution from the element to the PD is calculated as the first reflection power.

The contribution of the second and subsequent reflections are calculated using a modified Monte Carlo method based on ray tracing techniques. From each element, $N_{\text {ray }}$ rays with uniform distribution of equally likely random directions are generated. The emitted optical power of each ray becomes $P_{\text {ray-emit }}=P_{\text {ele-emit }} / N_{\text {ray }}$. When a ray reaches another element in the room, such an element again becomes a new point source to emit an optical power that depends on the reflection coefficient and the power contribution to the PD is then calculated. Each ray travels through the room and reflects off the surface of the room multiple times and its contribution to the PD from each reflection is collected. The process is repeated for all the rays and the reflection order can be as large as required. Basically, such a technique can be considered as a sequence of computing LOS contributions from a source to a receiver, both of which can be the reflecting element or the point at which the ray arrived.

Assuming that no optical concentrator is used at the receiver, the CIR from the LOS link between a source $S$ and a receiver $R$ can be written as

$h^{(0)}(t ; S, R)=\frac{\zeta_{R}^{S} P_{s}}{d_{S-R}^{2}} \cos ^{n} \phi \cos \psi \operatorname{rect}\left(\frac{\psi}{\Psi_{\frac{1}{2}}}\right) \delta\left(t-\frac{d_{S-R}}{c}\right)$, 


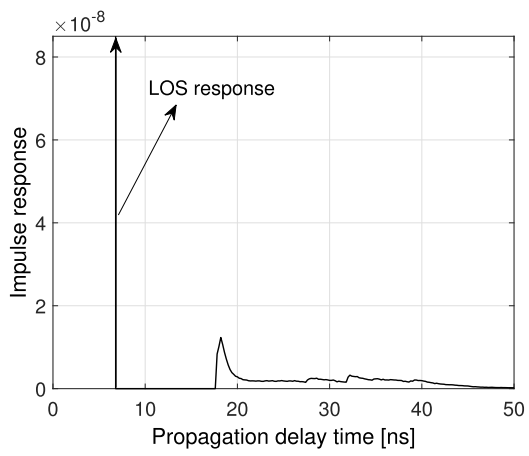

(a) when PD is at the point $\mathrm{A}$

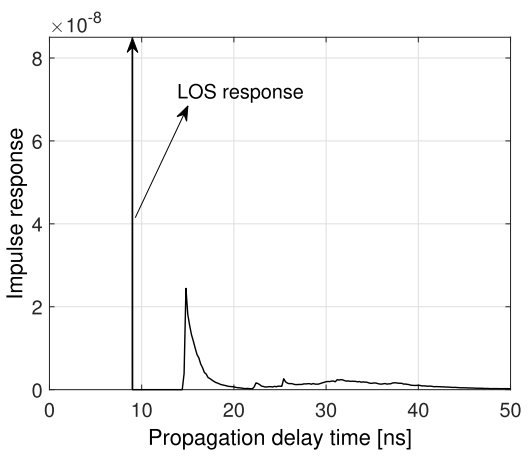

(b) when PD is at the point B

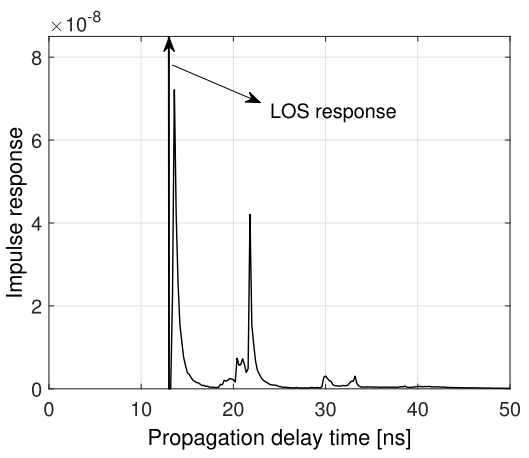

(c) when PD is at the point $\mathrm{C}$

Fig. 4. Impulse responses for three different receiver positions of $\mathrm{A}(2.45,2.45,1), \mathrm{B}(1.25,3.75,1)$ and $\mathrm{C}(0.15,4.85,1)$ as depicted in Fig. 3 .

where $d_{S-R}$ is the distance from the source to the receiver, $\phi$ is the angle of irradiance between the normal vector of the source and the direction of the emitted ray to the receiver. $\psi$ is the angle of incidence between the normal of the receiver and the direction of the incoming ray from the source, $\Psi_{\frac{1}{2}}$ is the field-of-view angle (FOV) of the receiver, which is set to 70 degrees for the PD and 90 degrees for the reflecting elements, $c$ is the speed of light. The term $\zeta_{R}^{S}$ is defined as $\zeta_{R}^{S}=(n+1) \Delta A_{R} / 2 \pi$ where $\Delta A_{R}$ is the active area of the receiver, which is either the reflecting element area or the PD area of $1.0 \mathrm{~cm}^{2}, n$ is the order of the Lambertian emission of the source, and is given by the source's semi-angle at half power $\Phi_{\frac{1}{2}}$ as $n=-\log _{2} \cos \left(\Phi_{\frac{1}{2}}\right)$. When the source is the reflecting element $\Phi_{\frac{1}{2}}$ is set to 60 degrees wheares $\Phi_{\frac{1}{2}}$ of the LED is 70 degrees. The function rect(.) is the rectangular function, which fixes the FOV angle of the receiver defined as,

$$
\operatorname{rect}(x)= \begin{cases}1, & \text { for }|x| \leq 1 \\ 0, & \text { for }|x|>1\end{cases}
$$

Furthermore, $P_{s}$ is the normalized average optical transmit power of the source, which is set to $1 \mathrm{~W}$ when calculating the CIR from the LED to the receiver.

Figure 4 illustrates the simulated CIRs for three PD positions of $\mathrm{A}(2.45,2.45,1), \mathrm{B}(1.25,3.75,1)$ and $\mathrm{C}(0.15,4.85,1)$, which corresponds to the center, a mid-point between room center \& corner, and the corner of the room as illustrated in Fig. 3. The channel simulation parameters used are given in Table II. The area of each reflecting element is $10.0 \mathrm{~cm}^{2}$. The number of random rays generated at each reflecting element is $N_{\text {ray }}=20$. We present the results for reflections up to fourth-order since higher reflection orders have very small power contribution. As the user moves from the center to the corner of the room the LOS contribution is decreased from $6.53 \times 10^{-6}$ at point $\mathrm{A}$ to $2.28 \times 10^{-6}$ and $5.85 \times 10^{-7}$ at point $\mathrm{B}$ and $\mathrm{C}$, respectively, while the LOS delay is increased. The non-line-of-sight (NLOS) contribution is increased at the corner of the room in conjunction with a smaller time delay compared with the LOS path. More specifically, the peak response of the NLOS component in the center is about $1.27 \times 10^{-8}$ while this value at the corner is about 6 times larger than in the center. Therefore, ISI is expected to be
TABLE II

Channel Simulation Parameters

\begin{tabular}{lr}
\hline \hline \multicolumn{2}{c}{ Workspace configuration } \\
\hline Room dimensions $(L \times W \times H)$ & $5 \mathrm{~m} \times 5 \mathrm{~m} \times 3 \mathrm{~m}$ \\
Average reflection coefficient & 0.454 \\
FOV of reflecting elements & $90^{\circ}$ \\
Semi-angle of reflecting elements & $60^{\circ}$ \\
\hline \multicolumn{2}{c}{ LEDs } \\
\hline \multicolumn{2}{c}{ LED's coordinate (m) } \\
Semi-angle of the LED & $(2.5,2.5,3)$ \\
Maximum transmit power (W) & $70^{\circ}$ \\
\hline \multicolumn{2}{c}{ PDs } \\
\hline FOV angle of the PD & $70^{\circ}$ \\
PD active area (cm $\left.{ }^{2}\right)$ & 1.0 \\
Distance from PDs to floor (m) & 1.0 \\
PD responsivity (A/W) & 0.54 \\
\hline \hline
\end{tabular}

larger when user moves towards the corner where the LOS component is small and NLOS contribution is high.

\section{Theoretical Performance AnAlysis OF THE ECSAM SCHEME}

In this section, we describe how ECSAM symbols are detected at the receiver by proposing first an optimal and second a sub-optimal detection algorithm. The upper bound on symbol error rate (SER) is then derived analytically for the case of a LOS optical wireless channel, which is then used to estimate the bit error rate (BER). For system simplification, it is assumed that perfect symbol and slot synchronization is achieved. We consider transmitting $\mathcal{V}$ ECSAM symbols consecutively. Each symbol is composed of an $N$ chip sequence, which satisfies the constraints in (11). The length- $\mathcal{V} N$ chip sequence of $\mathcal{V}$ symbols can be written as $\mathbf{c}=\left\{\mathbf{x}_{1}, \ldots . \mathbf{x}_{\mathcal{V}}\right\}$ where the $v$ th symbol $\mathbf{x}_{v}$ is a length- $N$ sequence $\mathbf{x}_{v}=\left\{a \kappa_{1}^{v}, \ldots, a \kappa_{N}^{v}\right\}$. Consequently, the sequence c of $\mathcal{V}$ transmitted ECSAM symbols can be expressed as

$$
\mathbf{c}=\left\{a \kappa_{1}^{1}, \ldots, a \kappa_{N}^{1}, \ldots, a \kappa_{1}^{v}, \ldots, a \kappa_{N}^{v}, \ldots, a \kappa_{1}^{\mathcal{V}}, \ldots, a \kappa_{N}^{\mathcal{V}}\right\}
$$

The ECSAM waveform is prepared for transmission by passing the sequence $\mathbf{c}$ through a unit-amplitude rectangular 
pulse-shaping filter $g(t)$ of duration $T_{p}$ as

$$
u(t)=\sum_{i=1}^{\mathcal{V}} \sum_{l=N(i-1)+1}^{i N} c_{l} g\left(t-l T_{p}\right),
$$

where $c_{l}=a \kappa_{l-N(v-1)}^{v}$ represents the lth element of $\mathbf{c}, v$ is the symbol containing $c_{l}$, which is given as $v=\left\lceil\frac{l}{N}\right\rceil$.

At the receiver, the signal is passed through a matched filter (MF) employing a unit-energy filter $p(t)$ that is matched to $g(t)$ at the transmitter and then sampled at a rate $1 / T_{p}$ samples/s. With the effect of the CIR $h(t)$, the receiver matched-filter output at the PD can be expressed as

$$
y(t)=\mathcal{R} \sum_{i=1}^{\mathcal{V}} \sum_{l=N(i-1)+1}^{i N} c_{l} f\left(t-l T_{p}\right)+n(t),
$$

where $f(t)=g(t) * h(t) * p(t)$ is the combined impulse response of the channel, the transmitter and the receiver filters. The noise component $n(t)$ is an additive white Gaussian noise (AWGN) at the receive filter output, while $\mathcal{R}$ is the PD's responsivity measured in ampere/watt.

On a channel with multipath propagation, the optical signal from a symbol slot may spread beyond that symbol duration resulting in both interslot-interference and intersymbolinterference. The sample value of the $q$ th slot in the presence of noise can be expressed as (see Appendix for derivation)

$$
\begin{array}{r}
r_{v, q}=\mathcal{R} f_{0} a \kappa_{q}^{v}+\mathcal{R} \sum_{i=1}^{v} \sum_{\substack{l=N(i-1)+1 \\
l \neq N(v-1)+q}}^{i N} a \kappa_{l-N(v-1)}^{v} f_{N(v-1)+q-l} \\
+w_{v, q} . \quad \text { (18) }
\end{array}
$$

The first term in (39) represents the desired information signal while the second term represents the interslot and intersymbol interference, which is denoted as ISI in this paper. In the following section, the analytical performance analysis of ECSAM is derived for the LOS channel, that is, the ISI component is assumed to be zero and the received sample value $r_{v, q}$ is simplified to $r_{v, q}=\mathcal{R} h_{0} a \kappa_{q}^{v}+w_{v, q}$, where $f_{0}$ is reduces to LOS channel coefficient $h_{0}$. The effect of multipath dispersion is analyzed by computer simulation only in Section IV.

\section{A. Optimum Receiver}

In this subsection, we formulate the ML detector as the optimum receiver for the ECSAM scheme that decodes symbols using the channel state information estimated at the receiver. In this respect, it is reasonable that training symbols with all slots activated at full power are sent within the channel coherence time, such that the receiver can estimate the channel. Before detailing the detection procedure we briefly describe how the channel estimation procedure is carried out. $W$ number of pilot symbols, each of $T_{s}$ duration, are transmitted every $L T_{s}$ seconds, that is, every $L$ macro-symbols. The value of $L$ is chosen to guarantee that $L T_{s}$ is much smaller than the channel coherence time. The value of $W$ determines the accuracy of the channel estimate. The pulse sent for channel estimation is given by

$$
x_{e}(t)=\sum_{i=1}^{W} \sum_{l=N(i-1)+1}^{i N} \operatorname{Mag}\left(t-l T_{p}\right) .
$$

Then the received signal vector for channel estimation is given by

$$
y_{e}(t)=\mathcal{R} x_{e}(t) * h(t)+n(t) .
$$

By integrating the signal in (20) we can estimate the channel based on the minimum mean square error criterion (MMSE) so as to obtain the channel estimate $\tilde{h}_{0}$ as [20]

$$
\tilde{h}_{0}=\frac{1}{\mathcal{R} M a W} \sum_{m=1}^{W} \int_{T_{p}} y_{e}\left(t-m T_{s}\right) d t .
$$

For detection, the receiver should analyze the conditional probability of $\mathbf{r}_{v}$ on the $N$-length sequence for all possible symbols transmitted, which is a set given by the symbol combinations in (6), $\mathbb{S}_{z, M}^{N}$. The ML criterion can be described by

$$
\hat{\mathbf{r}}_{v}=\underset{\mathbf{x} \in \mathbb{S}_{z, M}^{N}}{\operatorname{argmax}} p\left(\mathbf{r}_{v} \mid \mathcal{R} \tilde{h}_{0} \mathbf{x}\right),
$$

where $p$ is the conditional probability. Under the hypothesis, largely verified, of zero mean $\mathcal{N}_{0}$-variance white Gaussian distributed noise, (22) can be expressed as

$$
p\left(\mathbf{r}_{v} \mid \mathcal{R} \tilde{h}_{0} \mathbf{x}\right)=\frac{1}{\left(2 \pi \mathcal{N}_{0}\right)^{N / 2}} \exp \left(-\frac{1}{2 \mathcal{N}_{0}}\left\|\mathbf{r}_{v}-\mathcal{R} \tilde{h}_{0} \mathbf{x}\right\|^{2}\right)
$$

The above expression leads to a comparison among all the selected combinations, which can be carried out by determining the minimum distance as

$$
\hat{\mathbf{x}}=\underset{\mathbf{x} \in \mathbb{S}_{z, M}^{N}}{\operatorname{argmin}}\left\|\mathbf{r}_{v}-\mathcal{R} \tilde{h}_{0} \mathbf{x}\right\|^{2} .
$$

Since the solution of (24) is essentially the $N$-tuple of symbols, the number of combinations may be very high. Thus, for very large values of $N$ ML detection becomes computationally prohibitive with implementation costs of the order of $\mathcal{O}(\mathcal{Q})$. For example, with $N=7, M=8$ and dimming level $z=0.5$ the number of combinations possible is $\mathcal{Q}=$ 273127. However, when $N$ increase from 7 to 8 , the number of combinations possible increases to $\mathcal{Q}=2306025$. This exponential increase in $\mathcal{Q}$ for moderate values of $N$ quickly makes ML detection computationally prohibitive. In the next section, we present a sub-optimal receiver for ECSAM that reduces the computational cost.

\section{B. Sub-Optimal Receiver}

A sub-optimal Rx is proposed in this section as an alternative to the computationally complex ML Rx. In the first step of the sub-optimal Rx, a slot-by-slot (that is one shot) detection is performed every $T_{p}$ seconds, where the amplitude index in the $i$ th slot of the $v$ th symbol can be estimated as

$$
\hat{\kappa}_{i}^{v}=\underset{\kappa_{i}^{v} \in\{0, \ldots, M\}}{\operatorname{argmin}}\left\|r_{v, i}-\mathcal{R} \tilde{h}_{0} a \kappa_{i}^{v}\right\|^{2},
$$


that is essentially the same detector in PAM demodulation. The estimated symbol after the slot-by-slot detection becomes $\hat{\mathbf{x}}_{v}=a \hat{\boldsymbol{\kappa}}^{v}$, where $\hat{\boldsymbol{\kappa}}^{v}=\left\{\hat{\kappa}_{1}^{v}, \cdots, \hat{\kappa}_{i}^{v}, \cdots, \hat{\kappa}_{N}^{v}\right\}$ is the estimated amplitude indices as presented in (25). However, detection only on the criterion in (25) does not fully account for the dimming constraint. Since the optical energy constraint is known both at the transmitter and the receiver, the latter can perform a check to determine if the dimming constraint is met at the Rx. If this is verified, it is reasonable to assume that the detection is error free, otherwise some correction mechanism can be applied.

The total optical energy of the detected sequence $\hat{\mathbf{x}}_{v}$ can be calculated as

$$
\mathcal{E}_{d}^{o}=a T_{p} \sum_{i=1}^{N} \hat{\kappa}_{i}^{v}
$$

We define $\Delta$ as the measure of distance between the optical energy of the detected sequence $\hat{\mathbf{x}}_{v}$ and that specified by the dimming constraint as

$$
\Delta=\frac{\mathcal{E}_{d}^{o}-\mathcal{E}^{o}}{a T_{p}},
$$

such that (27) can be expressed as

$$
\Delta=\sum_{i=1}^{N}\left(\hat{\kappa}_{i}^{v}-\kappa_{i}^{v}\right) .
$$

Equation (28) essentially measures the difference between the detected and transmitted amplitude indices for the transmitted symbol $\mathbf{x}_{v}$. There are three possible outcomes for $\Delta$ : zero, positive or negative. Depending on each outcome the receiver will adopt different processes.

For $\Delta=0$, the receiver will assume that the detection is error free. However, it is possible, for example, that the difference is zero for each $i$ value with the exception of two $i$ values where $\hat{\kappa}_{i}^{v}-\kappa_{i}^{v}=1, \hat{\kappa}_{i}^{v}-\kappa_{i}^{v}=-1$. In this case, we have two errors, which the receiver is unable to detect since only the sum energy is calculated.

For $\Delta>0$ the detection is affected by at least $\Delta$ errors. In fact, for this case the value of $\Delta$ does not indicate the exact number of errors for two reasons. The first one is related to the error-distance mechanism described when $\Delta=0$. The second reason is that the receiver might select a PAM-symbol with distance $\hat{\kappa}_{i}^{v}-\kappa_{i}^{v}>1$ for the $i$ th slot. This second case tends to happen at very low values of SNR, when noise dominates. Hence, when $\Delta>0$ it is assumed that the one shot detector in (25) selects the ECSAM sequence element that has more energy than expected. To solve this, a correction mechanism is used after the one shot detector to correct the incorrectly estimated ECSAM slots and further minimise the errors. Denote $\mathbf{p}$ as the non-zero entries of $\hat{\boldsymbol{\kappa}}^{v}$ and $\mathbf{q}$ as the corresponding received amplitudes in $\mathbf{r}_{v}$. If the number of non-zero detected slots $|\mathbf{p}| \leq \Delta$, then the detected non-zero amplitude indices are reduced by $a \frac{\Delta}{|\mathbf{p}|}$ for the case $\Delta$ is divisible by $|\mathbf{p}|$ and by $a\left\lfloor\frac{\Delta}{|\mathbf{p}|}\right\rfloor$ in case it's not. The latter requires repeating the correction process from the beginning to correct the remaining errors. For the case $|\mathbf{p}|>\Delta$, the correction mechanism checks the squared distance $\mathbf{v}$ (later referred to as distance $\mathbf{v}$ ) from each time slot with lower amplitude values as

$$
\mathbf{v}=\left\|\mathbf{q}-\mathcal{R} \tilde{h}_{0} a(\mathbf{p}-\mathbf{1})\right\|^{2},
$$

Non-zero time slots with smaller distances in $\mathbf{v}$ are more likely overestimated in the one shot detector which leads to higher symbol energy. Therefore, using the index of the lowest distances in $\mathbf{v}$ the amplitude of the corresponding slots within $\hat{\boldsymbol{\kappa}}^{v}$ is reduced by 1 to estimate the symbol with correct energy.

For $\Delta<0$, the procedure used when $\Delta>0$ remains valid since when $\Delta<0$ at least $\Delta$ errors are incurred so a mechanism similar to $\Delta>0$ can be used. Denote the slots with detected amplitude indices lower than the maximum value $M$ as $\overline{\mathbf{p}}$ and their corresponding received signal in $\mathbf{r}_{v}$ as $\overline{\mathbf{q}}$. First, the correction mechanism checks if $|\overline{\mathbf{p}}| \leq|\Delta|$, then detected non-maximum amplitude indices will be increased by $a \frac{|\Delta|}{|\overline{\mathbf{p}}|}$ for the case $\Delta$ is divisible by $|\overline{\mathbf{p}}|$ and by $a\left\lfloor\frac{|\Delta|}{|\overline{\mathbf{p}}|}\right\rfloor$ for the case it's not. The latter requires repeating the correction process from the beginning to correct the remaining errors. For the case $|\overline{\mathbf{p}}|>|\Delta|$, the correction mechanism checks the squared distance $\mathbf{w}$ (later referred to as distance $\mathbf{w}$ ) for each time slot with higher amplitude values as

$$
\mathbf{w}=\left\|\overline{\mathbf{q}}-\mathcal{R} \tilde{h}_{0} a(\overline{\mathbf{p}}+\mathbf{1})\right\|^{2},
$$

Non-maximum time slots with smaller distances in $\mathbf{w}$ are more likely to be underestimated by the one shot detector, which leads to lower symbol energy. Therefore, using the index of the lowest distances in $\mathbf{w}$ the amplitude of the corresponding slots within $\hat{\boldsymbol{\kappa}}^{v}$ is increased by 1 to estimate the symbol with correct energy.

Example 2: Let's consider the ECSAM waveforms in Table I, the LOS channel coefficient of 1, and PD responsivity of $1(\mathrm{~A} / \mathrm{W})$. Assuming that the received marco-symbol is $\mathbf{r}=\{0.9,0.1,0.08,0.21\}$. After the slot-by-slot detection in (25), the estimated symbol is $\hat{\mathbf{x}}=\{1,0,0,0.25\}$. With the energy check in (28), we have $\Delta=1$, which means the slot-by-slot detector estimated the symbol with higher energy than expected. With correction mechanism described above, we have $\mathbf{p}=\{1,0.25\}$ and $\mathbf{q}=\{0.9,0.21\}$. The distance $\mathbf{v}$ can be calculated from (29) as $\mathbf{v}=\{0.0225,0.0441\}$. Consequently, the corrected symbol is $\hat{\hat{\mathbf{x}}}=\{0.75,0,0,0.25\}$.

\section{C. "Coding" Effect and Union Bound}

The proposed scheme is expected to perform better in terms of error rate than PAM both when energy constrained (i.e. with dimming support) and unconstrained whereas it is expected to perform worse than PPM (and its variations like those presented in [7], [10], [12]). This is due to the fact that the number of combinations $\mathcal{Q}<(M+1)^{N}$ results in less than the maximum number of combination possible being used. This resembles a coded system where not all the combinations are used and the distance between $\mathcal{Q}$ and $(M+1)^{N}$ is a measure of the robustness of the ECSAM with respect to errors, which is known as error correction capability of a code. The reason why this scheme is expected to outperform PAM is that such a correction capability is not present in PAM. The constrained PAM, that is, PAM with a constrained energy 
value every $T_{s}$ seconds, leads to less distance between symbols so increasing the error rate. On the other hand, PPM presents a larger distance between symbols though its rate is lower. Hence, ECSAM represents a beneficial, flexible compromise, which can be viewed as a generalization of $N$-PPM and $(M+1)$-PAM. In fact, when $\mathcal{E}^{o}=a T_{p}$ we have $N$-PPM. If the constraint on $\mathcal{E}^{\circ}$ is removed we have $(M+1)$-PAM. The above comments particularly interesting if we resort to the evaluation of performance in terms of the upper bound for the optimal detection case. According to [21] we can derive a measure of performance by using the union bound as

$$
\begin{aligned}
p_{s}\left(e \mid \boldsymbol{\kappa}^{i}\right) & =\operatorname{Pr}\left\{\bigcup_{\substack{j=1 \\
j \neq u}}^{\mathcal{Q}} e_{j i}\right\} \leq \sum_{\substack{j=1 \\
j \neq u}}^{\mathcal{Q}} \operatorname{Pr}\left(e_{j i}\right) \\
& =\sum_{\substack{j=1 \\
j \neq i}}^{\mathcal{Q}} \frac{1}{2} \operatorname{erfc}\left(\frac{\mathcal{R} h_{0} a d_{i j}}{\sqrt{\mathcal{N}_{0}}}\right),
\end{aligned}
$$

where the term $d_{i j}=\left\|\boldsymbol{\kappa}^{i}-\boldsymbol{\kappa}^{j}\right\|$ measures the Euclidean distance between symbols $i$ and $j$.

Hence, the average bit error probability can be upper bounded by

$$
p_{b}(e) \leq \frac{1}{2 \mathcal{Q} \log _{2} \mathcal{Q}} \sum_{i=1}^{\mathcal{Q}} \sum_{\substack{j=1 \\ j \neq i}}^{\mathcal{Q}} d_{H}\left(\mathbf{n}^{i}, \mathbf{n}^{j}\right) \operatorname{erfc}\left(\frac{\mathcal{R} h_{0} a d_{i j}}{\sqrt{\mathcal{N}_{0}}}\right) .
$$

where $d_{H}\left(\mathbf{n}^{i}, \mathbf{n}^{j}\right)$ is the Hamming distance between the two binary symbols corresponding to the signal vectors $\mathrm{x}^{i}$ and $\mathbf{x}^{j}$. From the above relationship it is possible to infer that, as distinct from other modulation formats, where in principle all symbol combinations are possible, in ECSAM we have constraints when defining the macro-symbols and this reduces the number of possible combinations with respect to, for example, PAM. The expression in (31) is useful as a benchmark when we consider high $\mathcal{Q}$ values since we can evaluate the performance of the sub-optimal receiver and how the error rate degrades compared to the ML detector.

\section{Numerical Results}

In this section, we evaluate the performance of the proposed ECSAM scheme in terms of spectral efficiency and error probability compared with PAM and MPPM in both LOS and ISI channels. For dimming support, the same constraint on the maximum transmitted energy $\mathcal{E}_{\max }^{o}$ within the symbol time $T_{s}$ is applied for PAM. With the average transmit power of $z A$, the set of $M+1$ PAM amplitude levels at dimming target $z$ can be written as

$$
P_{m}^{\mathrm{PAM}}= \begin{cases}\frac{2 z A}{M}(m-1), & z \leq \frac{1}{2} \\ (2 z-1) A+\frac{2(1-z) A}{M}(m-1), & z>\frac{1}{2}\end{cases}
$$

where $m=1,2, \ldots, M+1$. One of the advantages of PAM is that the number of bits per symbol remains constant irrespective of the dimming levels. Thus PAM is able to transmit

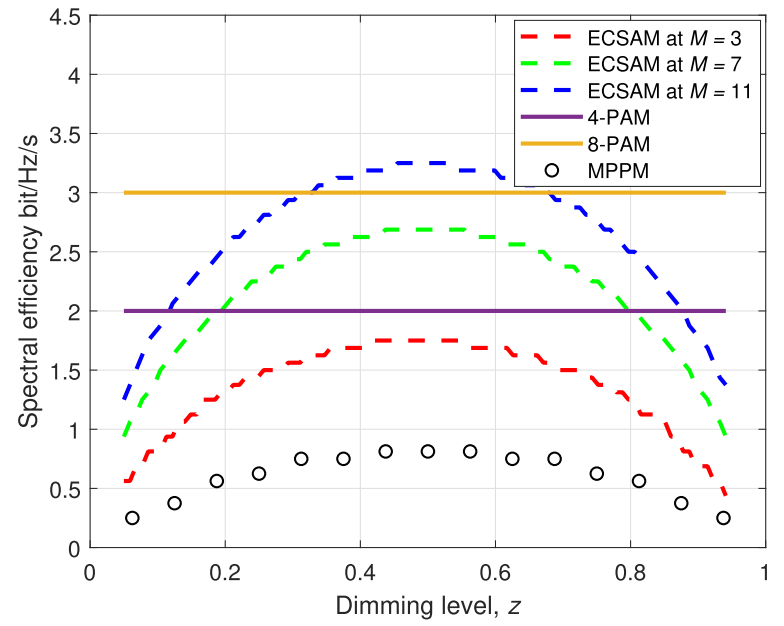

Fig. 5. Achievable spectral efficiency of ECSAM, PAM at different number of amplitude levels $M$ and MPPM as a function of dimming target. Number of slots is set at $N=16$.

data at very high rates compared with the other pulse-based modulation schemes. The achievable SE of PAM with $M+1$ amplitude levels is given by $\eta_{\mathrm{PAM}}=\left\lfloor\log _{2}(M+1)\right\rfloor$. In the case of MPPM, the dimming level can be adjusted by varying the number of active slots in a symbol. When $N_{a}$ slots are active, dimming level in MPPM can be calculated as $\frac{N_{a}}{N}$. The achievable SE of MPPM is given as $\eta_{\text {MPPM }}=\frac{\left\lfloor\log _{2}\left(\begin{array}{l}N \\ N_{a}\end{array}\right)\right\rfloor}{N}$.

The achievable SE $\eta(\mathrm{bit} / \mathrm{s} / \mathrm{Hz})$ of ECSAM, PAM and MPPM are compared in Fig. 5 for full-range of dimming. The number of time slots is fixed at $N=16$ for ECSAM and MPPM. For a fair comparison, SE of ECSAM is compared with that of $(M+1)$-PAM. The SE of ECSAM for the number of amplitude levels $M$ of 3, 7, and 11 are shown. With this configuration, MPPM supports 15 dimming levels from 1/16 to $15 / 16$ with a peak SE of $0.8125 \mathrm{bit} / \mathrm{s} / \mathrm{Hz}$ in the center of the dimming range. At low and high dimming levels, SE of MPPM reduces significantly. In case of ECSAM and PAM, the SE depends also on the number of amplitude levels $M$. As can be seen from Fig. 5, SE of PAM remains constant for all dimming levels. The achievable SE of 4-PAM and 8-PAM are $2 \mathrm{bit} / \mathrm{s} / \mathrm{Hz}$ and $3 \mathrm{bit} / \mathrm{s} / \mathrm{Hz}$, respectively. Similar to that of MPPM, SE's curve of ECSAM is symmetric where largest values are in the center of the dimming range while in the low and high dimming ranges SE decreases gradually.

When $M=3$, ECSAM is capable of providing a SE of more than $1 \mathrm{bit} / \mathrm{s} / \mathrm{Hz}$ for dimming ranging from 0.15 to 0.85 , with the peak SE of $1.75 \mathrm{bit} / \mathrm{s} / \mathrm{Hz}$ in the middle dimming range. When the number of amplitude levels increases to $M=7$ and $M=11$ as shown in Fig. 5, the peak SE of ECSAM is around 2.69 and $3.19 \mathrm{bit} / \mathrm{s} / \mathrm{Hz}$, respectively. At the same number of amplitude levels, the SE of PAM is slightly higher than ECSAM in the middle dimming range. The SE of ECSAM can be further increased by increasing the number of time slots $N$ at the cost of higher system complexity as the number of combinations increases exponentially. It should be noted that the values of $M$ and $N$ in ECSAM are unconstrained and can be any non-negative value, which makes ECSAM highly 


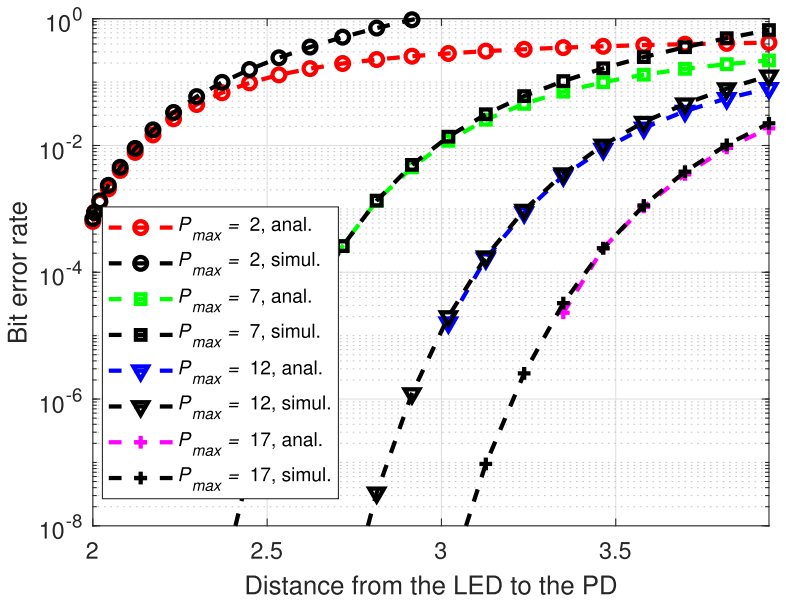

Fig. 6. Error performance of ECSAM when moving the PD from the center of the room to the corner at different transmit optical powers, the dimming level is fixed at $z=0.25$.

flexible. On the other hand, the number of amplitude levels in PAM is usually chosen to be a power of two.

In terms of error probability, we consider the system model presented in Section II-C with the channel simulation parameters in Table II. In this system, the PD suffers from shot noise and thermal noise.

In the case of strong background noise measured in daylight, the total noise variance can be calculated as [22] $\sigma^{2}=$ $C_{1} P_{r}+C_{2}$ where $C_{1}$ and $C_{2}$ depend mainly on the properties of the receiver design and the background noise, their values are adapted from [22] as $C_{1}=1.696 \times 10^{-11}$ and $C_{2}=$ $1.336 \times 10^{-13}$, and $P_{r}$ represents the desired received power. With the noise bandwidth of $B$, the noise power spectral density is given by $\mathcal{N}_{0}=\frac{2 \sigma^{2}}{B}$. The SNR can be defined as $\mathrm{SNR}=\frac{\mathcal{E}^{e}}{\mathcal{N}_{0}}$ where $\mathcal{E}^{e}$ is the average received symbol energy in the electrical domain at the PD. The signal waveform set with the highest Euclidean distance is denoted as $\mathbb{S}_{z}$, then the number of signal waveforms $\mathcal{Z}=\left|\mathbb{S}_{z}\right|$ should be a power of two for binary communications. The $k$ th waveform $(1 \leq k \leq \mathcal{Z})$ in the set $\mathbb{S}_{z}$ is denoted as $1 \times N$ vector $\mathbf{x}_{k}$. The average received symbol energy in the electrical domain for all the schemes can be express as

$$
\mathcal{E}^{e}=\frac{\sum_{k=1}^{\mathcal{Z}}\left\|\mathbf{x}_{k}\right\|^{2}}{\mathcal{Z}}\left(\mathcal{R} h_{0}\right)^{2} T_{p},
$$

where $h_{0}$ is the main desired path from the LED to the PD, $T_{p}$ is the pulse duration, which is equal to the symbol period $T_{s}$ in PAM and equal to the slot duration in ECSAM.

To evaluate the error performance of ECSAM, we first calculate the SNR value corresponding to the channel response $h$ at each PD position. The bit error rate is then calculated using both Monte-Carlo simulation and the analysis presented Section III. Fig. 6 depicts the bit error rate performance of ECSAM in the LOS channel when increasing the distance between the LED and PD by moving the PD from the room center towards the corner as depicted by the line A-C in Fig. 3. The error rate at different maximum transmit powers is also presented. The bandwidth is fixed at $100 \mathrm{MHz}$ for all the plots. The dimming level is fixed at $z=0.25$ with the number of

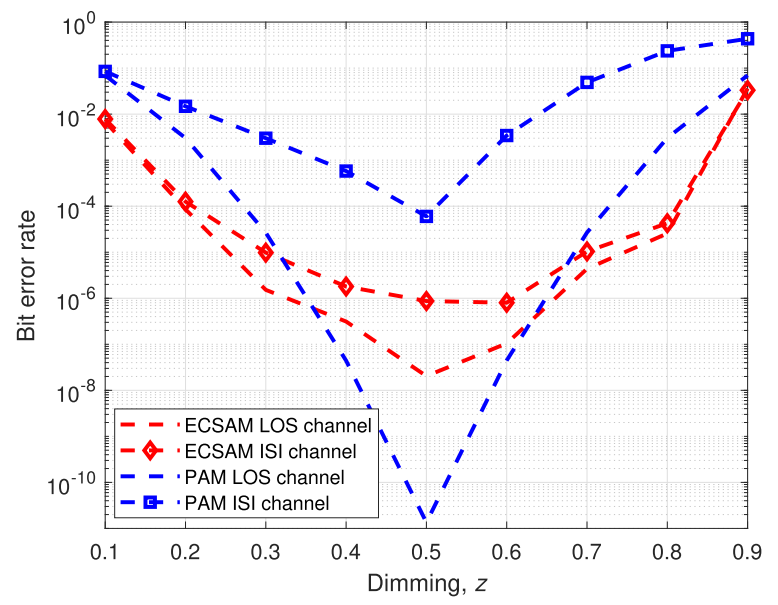

(a) Bit rate $100 \mathrm{Mbit} / \mathrm{s}$

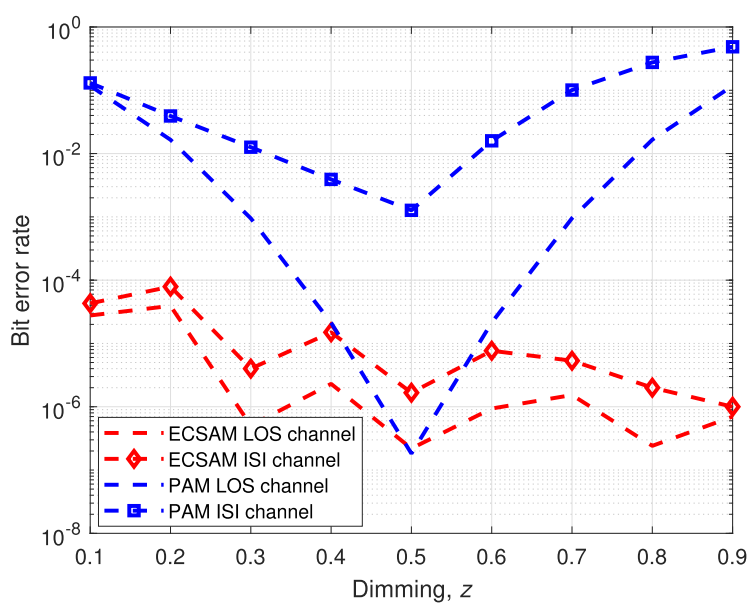

(b) Bandwidth $75 \mathrm{MHz}$

Fig. 7. Error performance comparison between ECSAM and PAM in both LOS and ISI channels as a function of dimming target for $N=4$ and $M=4$.

slots per symbol $N=4$ and the number of amplitude levels $M=4$, which results in an achievable bit rate of $125 \mathrm{Mbit} / \mathrm{s}$. There is a better match between the simulated BER and the upper bound results in the high SNR regime, as expected. At a maximum transmit power of 2 watts, the BER is high $\left(>10^{-3}\right)$ at a distance of 2 meters between the PD and LED. Acceptable BER is achieved when increasing $P_{\max }$ to 7 watts and higher. It is seen that with an amount of optical power from 7 to 17 watts, ${ }^{1}$ reliable light communication of $125 \mathrm{Mbit} / \mathrm{s}$ with BER lower than $10^{-6}$ can be achieved at a distance between the LED and PD of about 2.5 to $3.2 \mathrm{~m}$. When higher power is applied, the distance required to achieve a BER value is increased slightly. It must be noted that through the use of forward error correction (FEC), the error performance can be further improved.

Figure 7 illustrates the error performance comparison between ECSAM and PAM for both LOS and ISI channels over the full range of dimming. In this figure, the PD is

\footnotetext{
${ }^{1}$ It should be noted that in a practical scenario, use of multiple LEDs will reduce the required optical power. For example by placing an LED near the corner of the room shown in Fig. 3.
} 


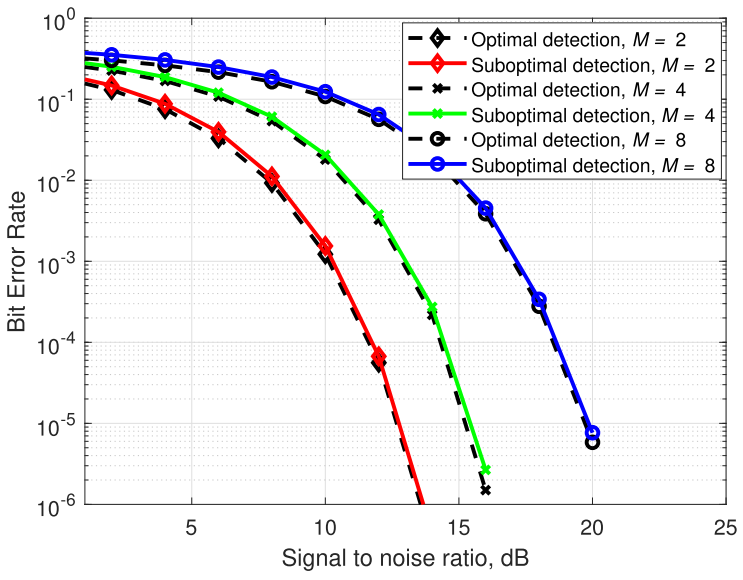

(a) LOS channel

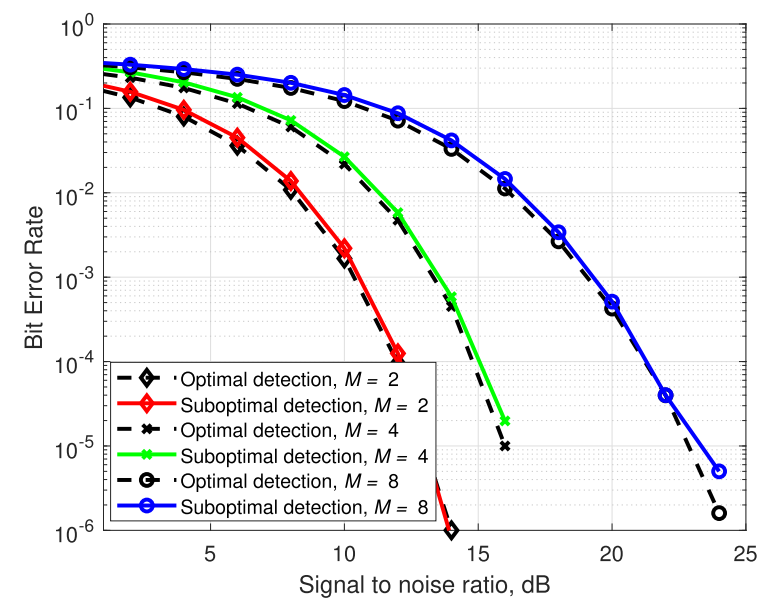

(b) ISI. channel

Fig. 8. Error performance comparing suboptimal detection with optimum ML detection at dimming level $z=0.25$ for both LOS and ISI channel when increasing $M$ from 2 to 8 and fixing $N=4$.

at position B with coordinates $(1.25,3.75,1)$ as depicted in Fig. 3, meaning that the distance between the PD and LED is around $2.6 \mathrm{~m}$. The channel response can be visualized from Fig. 4(b). As the SE of ECSAM is dependent on the dimming level whereas in PAM the SE is the same irrespective of the dimming, we present two results fixing the bit rate and then the bandwidth for both schemes to give a fair comparison. When fixing the bit rate of both schemes at $100 \mathrm{Mbit} / \mathrm{s}$ as shown in Fig. 7(a), the error rate at low and high dimming is significantly higher than in the centher of the dimming range for both ECSAM and PAM. In PAM, this can be explained as in the center of the dimming range the Euclidean distance between symbols is maximized. For ECSAM, the effect is due to the smaller number of symbols available at the extreme dimming regions compared with the middle dimming region and the Euclidian distance between each ECSAM symbol. As a result, a symmetric characteristic can be seen for PAM but not for ECSAM in the LOS channel.

According to Fig. 7(a), ECSAM provides better error performance than PAM in a LOS channel for dimming levels less than 0.36 and higher than 0.63 . In an ISI channel with fixed bit rate, the PAM scheme is significantly affected by ISI

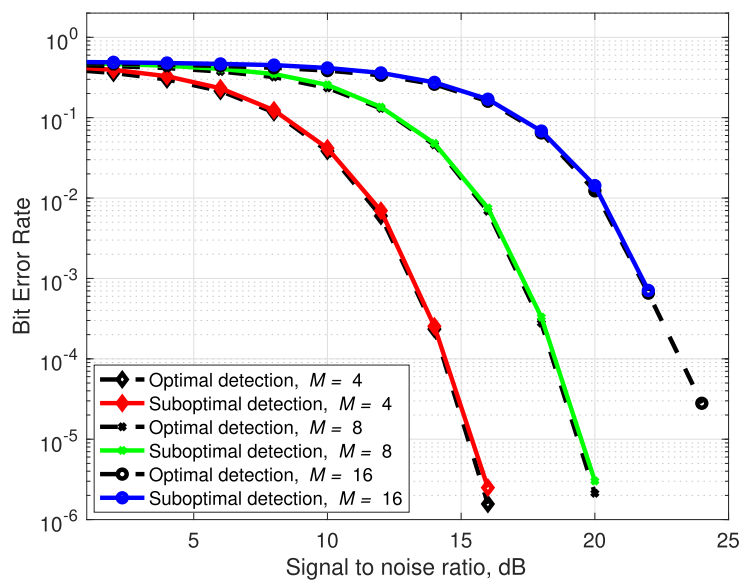

(a) LOS channel

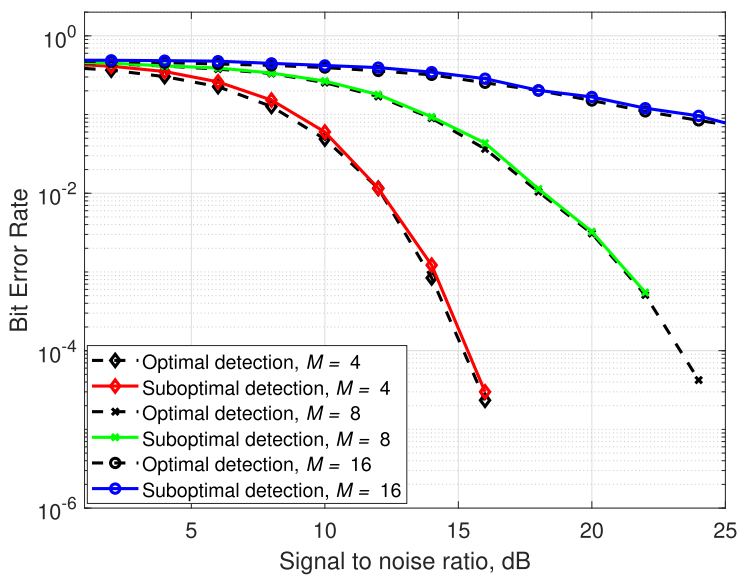

(b) ISI. channel

Fig. 9. Error performance comparing suboptimal detection with optimum ML detection at dimming level $z=0.25$ for both LOS and ISI channel when increasing $M$ from 4 to 16 and fixing $N=8$.

compared with ECSAM. More specifically, at a dimming level of 0.5 , the error rate of PAM increases from $1.3 \times 10^{-11}$ in a LOS channel to $6 \times 10^{-5}$ in an ISI channel whereas ECSAM is more immune to ISI as the error rate is at about $8 \times 10^{-7}$. This behavior can be explained by the energy constraint requirement in ECSAM. Since every ECSAM macro-symbol carries the same amount of optical energy, the possibility of having an error in the presence of ISI is significantly reduced compared with PAM where the constraint is only on the average energy of the data symbol. The effectiveness of ECSAM over PAM can be further demonstrated by fixing the bandwidth at $75 \mathrm{MHz}$ as shown in Fig. 7(b). The error rate of ECSAM is significantly lower than PAM for both LOS and ISI channel when using the same bandwidth. Furthermore, the error rate of ECSAM at low and high dimming becomes comparable with those in the center of the range when the same bandwidth is used for all the dimming levels. This suggests that ECSAM can be an adaptive modulation scheme for high illumination and communication performance VLC systems.

Besides the benefits of ECSAM with optimal detection in terms of SE and error performance, the large number of 


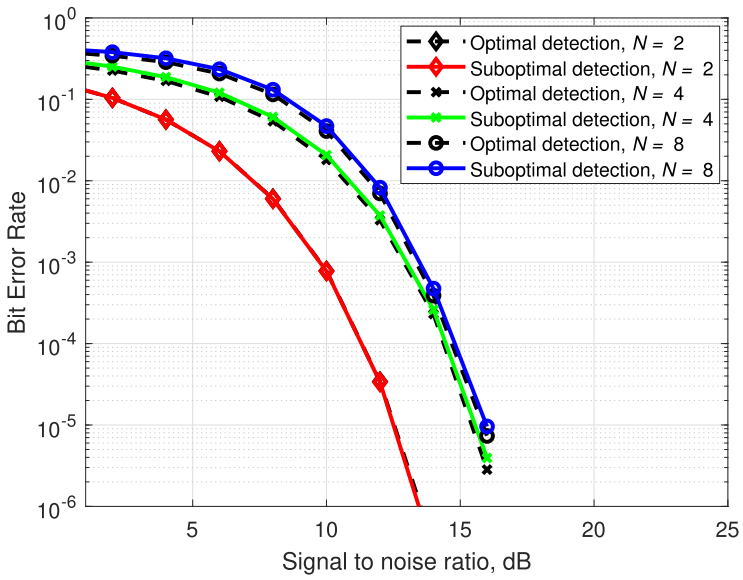

(a) LOS channel

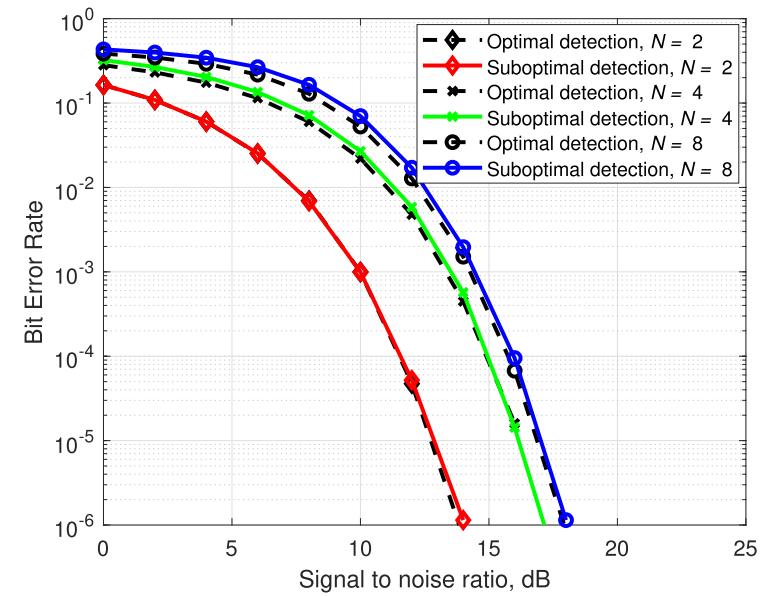

(b) ISI. channel

Fig. 10. Error performance comparing suboptimal detection with optimum ML detection at dimming level $z=0.25$ for both LOS and ISI channel when increasing $N$ from 2 to 8 and fixing $M=4$.

symbol combinations available leads to high computational complexity and cost of the receiver. In this respect, sub-optimal detection is proposed to offer less complexity to the system. In terms of error performance, Fig. 8 compares the BER of ECSAM for both LOS and ISI channels using optimal and sub-optimal detection for $N=4$ and $M=2,4$, and 8 . The same PD position of $(1.25 \mathrm{~m}, 3.75 \mathrm{~m}, 1 \mathrm{~m})$ is used at a bit rate of $100 \mathrm{Mbit} / \mathrm{s}$ and the dimming level is set at 0.25 . As can be seen in Fig. 8(a), the BER of ECSAM employing the sub-optimal detection algorithm is very close to that achieved with ML detection in the LOS channel. Employing a high number of amplitude levels can increase the SE of ECSAM at the cost of increasing the BER. The SNR required to achieve BER of $10^{-6}$ when increasing $M$ from 2 to 4 and 8 is about $2 \mathrm{~dB}$ and $5 \mathrm{~dB}$, respectively. This effect becomes even more clearer in the ISI channel with multipath propagation as seen in Fig. 8(b), where the gap is about $3 \mathrm{~dB}$ and $9 \mathrm{~dB}$ of SNR when increasing $M$ from 2 to 4 and 8 .

The results for high values of $N$ and $M$ are shown in Fig. 9, where $N=8$ is demonstrated when varying $M$ from 4 to 16 . As shown in Fig. 9(a), the performance of the sub-optimal receiver is very close to the ML detection at high values

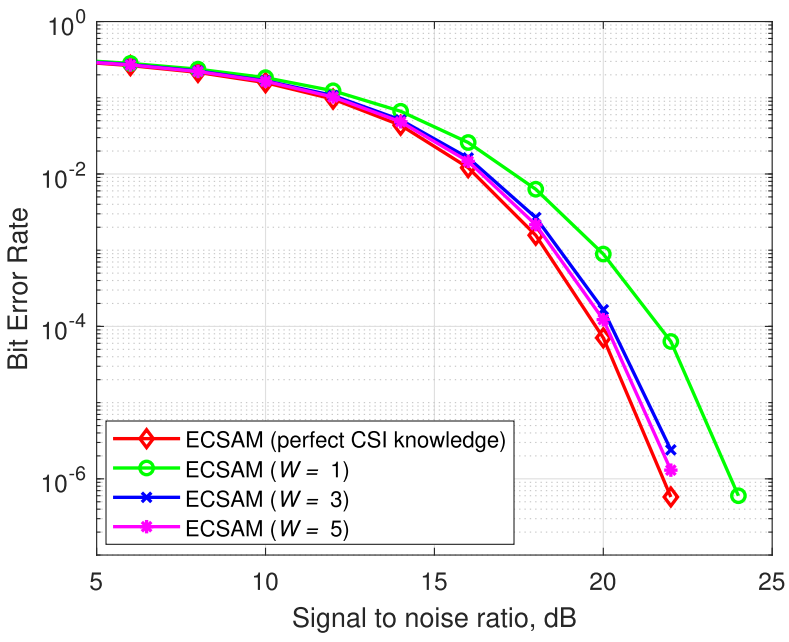

Fig. 11. The effect of channel estimation error on the error rate of ECSAM at dimming target $z=0.25$.

of $N$ and $M$ in the LOS channel. With $N=8$, the error penalty of increasing $M$ from 4 to 16 is clearer than for the case of $N=4$. In the ISI channel, the gap between the ML and sub-optimal receiver is increased at high values of $M$ as depicted in Fig. 9(b).

To further demonstrate the effectiveness of the proposed suboptimal detection, Fig. 10 illustrates the BER of ECSAM when increasing the number of time slots $N$ while keeping the number of amplitude level the same. In the LOS channel, Fig. 10(a) shows that increasing $N$ does not cause significant error performance degradation as compared with increasing $M$. More specifically, the SNR required to achieve BER of $10^{-6}$ when increasing $N$ from 2 to 4 and 8 is about $3 \mathrm{~dB}$ and $0.5 \mathrm{~dB}$, respectively. Furthermore, from Fig. 10(b), it is confirmed that the sub-optimal detection gives very good error performance when increasing both $N$ and $M$.

As ECSAM employs the maximum likelihood principle at the receiver, prior knowledge about the channel is a requirement. Therefore, it is interesting to see the effect of channel estimation on the BER performance. Let $W$ denote the number of symbols used for estimating the channel. Fig. 11 shows the effect of imperfect channel estimation in the LOS channel on the BER performance of ECSAM for a dimming level of 25\% and waveform configuration of $M=10, N=4$. When more symbols $W$ are employed to estimate the channel, the BER is significantly improved.

\section{CONCLUSION}

This paper proposed a low-complexity sub-optimal receiver architecture for a novel energy-constrained slot-amplitude modulation technique for VLC systems that uses both amplitude and time to modulate information while supporting dimming and flicker-free data transmission. A comprehensive performance comparison between the sub-optimal and maximum-likelihood ECSAM detectors has been presented along with performance comparison with other bandwidth and power efficient VLC modulation schemes. The investigations use line-of-sight and ISI (line-of-sight plus reflections) indoor VLC channel models for performance evaluation while varying 
the dimming levels. The bit error rate performance of the proposed receiver architecture is found to be very close to that of an ML detector. The investigations show that ECSAM provides complete flexibility in modifying the signal constellation for a desired dimming level to maximise the spectral efficiency and provide a robust BER performance when compared with conventional MPPM and PAM systems, especially in a hybrid channel.

\section{APPENDIX}

In order to obtain the signal for each slot, $y(t)$ is sampled at times $k T_{p}+\tau_{0}, k=1,2, \ldots$. Without loss of generality, assume that the transmission delay $\tau_{0}$ of the channel is zero, then we have the sample values as

$$
y\left(k T_{p}\right)=\mathcal{R} \sum_{i=1}^{\mathcal{V}} \sum_{l=N(i-1)+1}^{i N} c_{l} f\left(k T_{p}-l T_{p}\right)+n\left(k T_{p}\right),
$$

or, equivalently,

$$
y_{k}=\mathcal{R} \sum_{i=1}^{\mathcal{V}} \sum_{l=N(i-1)+1}^{i N} c_{l} f_{k-l}+n_{k}
$$

When the symbol $\mathbf{x}_{v}$ is received, the $1 \times N$ MF sample output vector for $N$ slots can be written as

$$
\mathbf{r}_{v}=\mathbf{s}_{v}+\mathbf{w}_{v}
$$

where $\mathbf{w}_{v}$ is the $1 \times N$ Gaussian noise vector at the sampling output, the $q$ th element of the $1 \times N$ vector $\mathbf{s}_{v}$ is given as

$$
s_{v, q}=\mathcal{R} \sum_{i=1}^{v} \sum_{l=N(i-1)+1}^{i N} a \kappa_{l-N(v-1)}^{v} f_{N(v-1)+q-l} .
$$

From (37) and (38), the sample value of the $q$ th slot in the presence of noise can be expressed as

$$
\begin{array}{r}
r_{v, q}=\mathcal{R} f_{0} a \kappa_{q}^{v}+\mathcal{R} \sum_{i=1}^{v} \sum_{\substack{l=N(i-1)+1 \\
l \neq N(v-1)+q}}^{i N} a \kappa_{l-N(v-1)}^{v} f_{N(v-1)+q-l} \\
+w_{v, q} \quad \text { (39) }
\end{array}
$$

where $w_{v, q}$ is zero-mean uncorrelated Gaussian random variables with a common variance of $\mathcal{N}_{0}$

\section{REFERENCES}

[1] Cisco Visual Networking Index: Global Mobile Data Traffic Forecast Update, Cisco, San Jose, CA, USA, Feb. 2017, pp. 2016-2021.

[2] H. Elgala, R. Mesleh, and H. Haas, "Indoor optical wireless communication: Potential and state-of-the-art," IEEE Commun. Mag., vol. 49, no. 9, pp. 56-62, Sep. 2011.

[3] A. Jovicic, J. Li, and T. Richardson, "Visible light communication: Opportunities, challenges and the path to market," IEEE Commun. Mag., vol. 51, no. 12, pp. 26-32, Dec. 2013.

[4] B. Lehman and A. J. Wilkins, "Designing to mitigate effects of flicker in LED lighting: Reducing risks to health and safety," IEEE Power Electron. Mag., vol. 1, no. 3, pp. 18-26, Sep. 2014.

[5] A. J. Shepherd, "Visual stimuli, light and lighting are common triggers of migraine and headache," J. Light Vis. Environ., vol. 34, no. 2, pp. 94-100, 2010.

[6] A. Tsiatmas, C. Baggen, F. Willems, J.-P. Linnartz, and J. Bergmans, "An illumination perspective on visible light communications," IEEE Commun. Mag., vol. 52, no. 7, pp. 64-71, Jul. 2014.
[7] S. Rajagopal, R. Roberts, and S.-K. Lim, "IEEE 802.15.7 visible light communication: Modulation schemes and dimming support," IEEE Commun. Mag., vol. 50, no. 3, pp. 72-82, Mar. 2012.

[8] K. Lee and H. Park, "Modulations for visible light communication with dimming control," IEEE Photon. Technol. Lett., vol. 23, no. 16, pp. 1136-1138, Aug. 15, 2011.

[9] J. Gancarz, H. Elgala, and T. Little, "Impact of lighting requirements on VLC systems," IEEE Commun. Mag., vol. 51, no. 12, pp. 34-41, Dec. 2013.

[10] M. Noshad and M. Brandt-Pearce, "Expurgated PPM using symmetric balanced incomplete block designs," IEEE Commun. Lett., vol. 16, no. 7, pp. 968-971, Jul. 2012.

[11] A. B. Siddique and M. Tahir, "Bandwidth efficient multi-level MPPM encoding decoding algorithms for joint brightness-rate control in VLC systems," in Proc. IEEE Global Commun. Conf., Dec. 2014, pp. 2143-2147.

[12] S. Pergoloni, M. Biagi, S. Rinauro, S. Colonnese, R. Cusani, and G. Scarano, "Merging color shift keying and complementary pulse position modulation for visible light illumination and communication," J. Lightw. Technol., vol. 33, no. 1, pp. 192-200, Jan. 1, 2015.

[13] J. K. Kwon, "Inverse source coding for dimming in visible light communications using NRZ-OOK on reliable links," IEEE Photon. Technol. Lett., vol. 22, no. 19, pp. 1455-1457, Oct. 1, 2010.

[14] K.-I. Ahn and J. K. Kwon, "Capacity analysis of M-PAM inverse source coding in visible light communications," J. Lightw. Technol., vol. 30, no. 10, pp. 1399-1404, May 15, 2012.

[15] T.-C. Bui, R. Singh, T. O'Farrell, and M. Biagi, "Energy-constrained slot-amplitude modulation with dimming support," IEEE Photon. Technol. Lett., vol. 30, no. 14, pp. 1301-1304, Jul. 15, 2018.

[16] T.-C. Bui, R. Singh, T. O'Farrell, and M. Biagi, "Performance evaluation of generalized optical spatial modulation with dimming support," in Proc. IEEE Globecom Workshops (GC Wkshps), Dec. 2018, pp. 1-6.

[17] M. Abramson, "Restricted combinations and compositions," Fibonacci Quart., vol. 14, no. 5, pp. 153-163, Dec. 1976.

[18] T. Komine and M. Nakagawa, "Fundamental analysis for visible-light communication system using LED lights," IEEE Trans. Consum. Electron., vol. 50, no. 1, pp. 100-107, Feb. 2004.

[19] M. I. S. Chowdhury, W. Zhang, and M. Kavehrad, "Combined deterministic and modified Monte Carlo method for calculating impulse responses of indoor optical wireless channels," J. Lightw. Technol., vol. 32, no. 18, pp. 3132-3148, Sep. 15, 2014.

[20] V. H. Poor, An Introduction to Signal Detection Estimation. New York, NY, USA: Springer, 1988.

[21] S. Benedetto and E. Biglieri, Principles of Digital Transmission: With Wireless Applications. Norwell, MA, USA: Kluwer Academic, 1999.

[22] T.-C. Bui, S. Kiravittaya, K. Sripimanwat, and N.-H. Nguyen, "A comprehensive lighting configuration for efficient indoor visible light communication networks," Int. J. Opt., vol. 2016, Dec. 2016, Art. no. 8969514 .

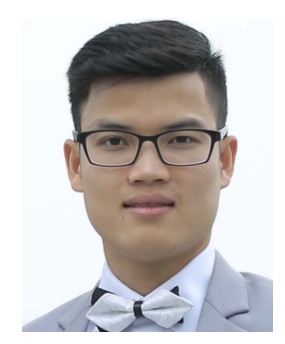

Thai-Chien Bui (Member, IEEE) received the M.Eng. degree in electrical engineering from Naresuan University, Thailand, in 2016, and the $\mathrm{Ph} . \mathrm{D}$. degree from the Department of Information, Electronics and Telecommunications Engineering, Sapienza University of Rome, Italy, in 2020. $\mathrm{He}$ is currently working as a Scientist Innovator with TNO, The Netherlands, where he is working on optical satellite communication systems. His research interests include physical layer techniques for visible light communication, free-space optical communication, and quantum key distribution.

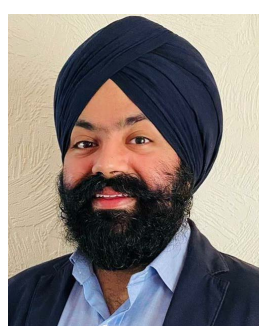

Ravinder Singh (Member, IEEE) received the M.Eng. and Ph.D. degrees from the Department of Electronic and Electrical Engineering, The University of Sheffield, U.K., in 2011 and 2016, respectively. He is currently a Post-Doctoral Research Assistant with the University of Oxford, where he is working on beam-steering and tracking terminals for fiber-wireless-fiber communication. He has a range of experience in RF and optical communication research in academia. His research interests include physical layer techniques for RF and optical communication systems, free-space optical links, and quantum communication. 
BUT et al: OPTICAL ENERGY-CONSTRAINED SLOT-AMPLITUDE MODULATION FOR DIMMABLE VLC

1595

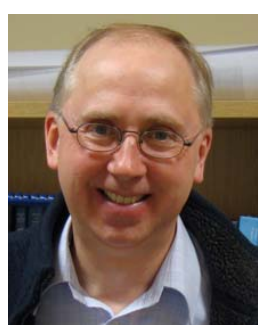

Timothy O'Farrell (Senior Member, IEEE) is currently a Professor in Wireless Communications with The University of Sheffield, U.K. He is an expert in wireless communication systems, specializing in physical layer signal processing, radio resource management, and wireless network planning. He has pioneered research on energy-efficient mobile eellular communications, the mathematical modeling of CSMA-based MAC protocols for $\mathrm{WiFi}$, iterative block coding for wireless communication systems, and spreading sequence design for CDMA wireless networks. He is an Entrepreneur, being the Co-Founder and CTO of Supergold Communication Limited (1997-2007), a start-up that participated in the standardization of IEEE 802.11g with the MBCK proposal. In the framework of Mobile VCE (mVCE), he was the Academic Coordinator of the Core 5 Green Radio project (2009-2012) and a leader in establishing energy efficiency as a global research field in wireless communications systems. Since 1996, he has managed 24 major research projects as a Principal Investigator (PI), with a total research spend of $£ 12.6 \mathrm{M}$. His current EPSRC project portfolio as a PI includes EP/S008101/1 (DD-mmMaMi), and previously EP/M013723/1 (FARAD); EP/L026147/1 (SERAN). He has published over 330 journals and conference papers, book chapters, patents, and technical reports; and has participated in standards, consultancy, and expert witness activities within the wireless sector. He was the General Chair of the Fth International Workshop on Next Generation Green Wireless Networks (Next-GWiN 2018) hosted by The University of Sheffield. Recently, he directed the U.K. Research Strategy Community Organisation in Communications, Mobile Computing and Networking (CommNet2) within the EPSRC portfolio (EP/N007824/1). $\mathrm{He}$ is the Director of the mICE, a Chartered Engineer, and an IET member.

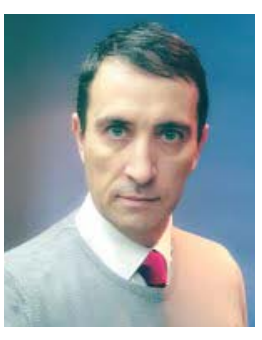

Mauro Bragi (Senior Member, IEEE) received the Laura degree in communication engineering and the $\mathrm{Ph} . \mathrm{D}$. degree in information and communicatimon theory from the Sapienza University of Rome, Rome, Italy, in 2001 and 2005, respectively. Since 2006, he has been serving as an Assistant Professor with the Department of Information, Electronics and Telecommunications Engineering, Sapienza Univercity of Rome, where he has been an Associate Professor since 2015. In 2010, he was a Visiting Assistant Professor with the Department of Electrical and Computer Engineering, The University of British Columbia, Vancouver, BC, Canada. He is responsible for the research activities in the field of visible light communications in the RGB-Comm Laboratory, where he advises Ph.D. students and postdocs leading projects in the area of optical wireless. His research interests include multiantenna systems, cognitive radio, underwater communications, smart grids, and ultrawideband. He leads the AMOUR-AQUALAB laboratory and projects regarding acoustic underwater communications. He is currently a member of the IEEE Communications Society Technical Committees on Power Line Communications, Cybersecurity and Transmission Access on Optical Systems. He has served as an Associate Editor for the IEEE Photonic TECHNOLOGY LETTERS.

Authorized licensed use limited to: Universita degli Stud di Roma La Sapienza. Downloaded on April 22,2021 at 08:10:39 UTC from IEEE Xplore. Restrictions apply. 